\title{
On $q$-hypergeometric Bernoulli polynomials and numbers
}

\author{
Salifou Mboutngam ${ }^{1}$ (D), Patrick Njionou Sadjang ${ }^{2 *}$ (D) \\ ${ }^{1}$ Higher Teachers' Training College, University of Maroua, Maroua, Cameroon \\ ${ }^{2}$ National Higher Polytechnic School of Douala, University of Douala, Douala, Cameroon
}

\begin{abstract}
We introduce $q$-analogues of the hypergeometric Bernoulli polynomials in one and two real parameters and study several of their properties. Also we provide the inversion, the power representation, the multiplication and the addition formula for these polynomials. Classical results are recovered by limit transition.
\end{abstract}

Mathematics Subject Classification (2020). 11B68, 05A30, 33D15

Keywords. $q$-Bernoulli polynomials, $q$-Bernoulli numbers, $q$-hypergeometric Bernoulli polynomials, recurrence relation, $q$-difference equation

\section{Introduction}

The Appell polynomials $A_{n}(x)$ defined by

$$
f(t) \mathrm{e}^{x t}=\sum_{n=0}^{\infty} A_{n}(x) \frac{t^{n}}{n !}
$$

where $f$ is a formal power series in $t$, have found remarkable applications in different branches of mathematics, theoretical physics and chemistry $[3,4,11,19,20]$. A special case of Appell polynomials are Bernoulli polynomials $B_{n}(x)$, generated by $f(t)=\frac{t}{\mathrm{e}^{t}-1}$ in (1.1). Also, Bernoulli numbers $B_{n}:=B_{n}(0)$ are of considerable importance in number theory, combinatorics and numerical analysis. They are represented as

$$
\frac{t}{\mathrm{e}^{t}-1}=\sum_{n=0}^{\infty} B_{n} \frac{t^{n}}{n !} \quad(|t|<2 \pi)
$$

or by the recurrence relation

$$
\sum_{k=0}^{n}\left(\begin{array}{c}
n+1 \\
k
\end{array}\right) B_{k}=0 \text { for } n \geq 1 \text { and } B_{0}=1 .
$$

\footnotetext{
*Corresponding Author.

Email addresses: mbsalif@gmail.com (S. Mboutngam), pnjionou@yahoo.fr (P. Njionou Sadjang)

Received: 04.01.2020; Accepted: 21.03.2021
} 
Bernoulli numbers are directly related to several combinatorial numbers such as Stirling, Cauchy and harmonic numbers. For example, except $B_{1}$ we have

$$
B_{n}=(-1)^{n} \sum_{m=0}^{n} \frac{(-1)^{m} m !}{m+1} S_{2}(n, m),
$$

where

$$
S_{2}(n, m)=\frac{1}{m !} \sum_{j=0}^{m}(-1)^{j}\left(\begin{array}{c}
m \\
j
\end{array}\right)(m-j)^{n},
$$

denote the second kind of Stirling numbers [5,9] with $S_{2}(n, m)=0$ for $n<m$.

These polynomials have found various extensions such as poly-Bernoulli numbers, which are somehow connected to multiple zeta values. Al-Salam [1] introduced the first qextension of Bernoulli numbers and polynomials and gave many of their properties. The $q$-extensions of Bernoulli numbers and polynomials have now found many applications in combinatorics statistics and various branches of applied mathematics.

In [12], F. T. Howard considers the following generalization of Bernoulli polynomials

$$
\frac{t^{2} e^{x t} / 2}{e^{t}-1-t}=\sum_{n=0}^{\infty} A_{n}(x) \frac{t^{n}}{n !}
$$

and more generally in [13], he considers

$$
\frac{t^{N} e^{x t} / N !}{e^{t}-T_{N-1}(t)}=\sum_{n=0}^{\infty} B_{n}(N, x) \frac{t^{n}}{n !}
$$

where

$$
T_{N}(t)=\sum_{n=0}^{N} \frac{t^{n}}{n !},
$$

and $N$ is any positive integer. For $N=1$ and $N=2,(1.3)$ reduces to (1.1) and (1.2), respectively.

The aim of this work is to introduce $q$-analogues of the hypergeometric Bernoulli polynomials and numbers. The paper is organised as follows: Section 2 provides some preliminary definitions and results useful for the reader. In Section 3, two $q$-analogues of the Hypergeometric Bernoulli polynomials and numbers are introduced and several of their properties are stated and proved. It is proved for example in Theorem 3.6 that the $q$-hypergeometric Bernoulli polynomials of the first kind are the only $q$-Appell set with zero moments. In Section 4, the $q$-hypergeometric Bernoulli polynomials with two real parameters are introduced, Section 5 studies some multiplication formulas and Section 6 introduces $q$-hypergeometric Bernoulli polynomials of higher order.

\section{Preliminary definitions and results}

The following definitions can be found in [17]. Let $n$ be a non-negative integer. The so-called $q$-number is defined by

$$
[n]_{q}=\frac{1-q^{n}}{1-q} .
$$

For a non-negative integer $n$, the $q$-factorial is defined by

$$
[n]_{q} !=\prod_{k=0}^{n}[k]_{q} \quad \text { for } \quad n \geq 1, \quad \text { and } \quad[0]_{q} !=1 .
$$

The $q$-binomial coefficients are defined by

$$
\left[\begin{array}{l}
n \\
k
\end{array}\right]_{q}=\frac{[n]_{q} !}{[k]_{q} ![n-k]_{q} !}, \quad(0 \leq k \leq n) .
$$


The following so-called $q$-Pochhammer numbers $(a ; q)_{n}$ are defined by

$$
(a ; q)_{0}=1, \quad(a ; q)_{n}=\prod_{k=0}^{n-1}\left(1-a q^{k}\right), \quad(n \geq 1) .
$$

It is not difficult to see that

$$
\left[\begin{array}{c}
n \\
k
\end{array}\right]_{q}=\frac{(q ; q)_{n}}{(q ; q)_{k}(q ; q)_{n-k}}, \quad(0 \leq k \leq n)
$$

For $n=\infty$ we set

$$
(a ; q)_{\infty}=\prod_{n=0}^{\infty}\left(1-a q^{n}\right),|q|<1 .
$$

From the definition of $(a ; q)_{\infty}$, it follows that for $0<|q|<1$, and for a nonnegative integer $n$, we have

$$
(a ; q)_{n}=\frac{(a ; q)_{\infty}}{\left(a q^{n} ; q\right)_{\infty}} .
$$

This enables an extension of the $q$-Pochhammer to any arbitrary complex number $\lambda$ by

$$
(a ; q)_{\lambda}=\frac{(a ; q)_{\infty}}{\left(a q^{\lambda} ; q\right)_{\infty}}, \quad 0<|q|<1
$$

where the principal value of $q^{\lambda}$ is taken.

The following notation

$$
(x \ominus y)_{q}^{n}=(x-y)(x-q y) \cdots\left(x-q^{n-1} y\right),
$$

is called the $q$-power basis. It generalises the power and the $q$-pochhammer since

$$
(1 \ominus y)_{q}^{n}=(y ; q)_{n} \quad \text { and } \quad(x \ominus 0)_{q}^{n}=x^{n} .
$$

We will use the following two $q$-analogues of the exponential function $e^{x}$ :

$$
e_{q}(x)=\sum_{k=0}^{\infty} \frac{x^{k}}{[k]_{q} !}
$$

and

$$
E_{q}(x)=\sum_{k=0}^{\infty} \frac{q^{\left(\begin{array}{c}
k \\
2
\end{array}\right)}}{[k]_{q} !} x^{k}
$$

These two functions are related by the equation (see [15])

$$
e_{q}(x) E_{q}(-x)=1 \text {. }
$$

Remark 2.1. It is not difficult to see that $[17$, Eq. (1.14.1) and (1.14.2)]

$$
\begin{aligned}
& e_{q}(x)=\frac{1}{((1-q) x ; q)_{\infty}}, \quad 0<|q|<1, \quad|z|<1 \\
& E_{q}(x)=(-(1-q) x ; q)_{\infty}, \quad 0<|q|<1 .
\end{aligned}
$$

Definition 2.2 ( $q$-addition, see [15]). Let $x$ and $y$ be two complex numbers and $n$ a nonnegative integer. We define the $q$-addition in the following way

$$
\left(x \oplus_{q} y\right)^{n}:=\sum_{k=0}^{n}\left[\begin{array}{l}
n \\
k
\end{array}\right]_{q} x^{k} y^{n-k} .
$$

Definition 2.3 (See [8]). The basic hypergeometric or $q$-hypergeometric function ${ }_{r} \phi_{s}$ is defined by the series

$$
{ }_{r} \phi_{s}\left(\begin{array}{c}
a_{1}, \cdots, a_{r} \\
b_{1}, \cdots, b_{s}
\end{array} \mid q ; z\right):=\sum_{k=0}^{\infty} \frac{\left(a_{1}, \cdots, a_{r} ; q\right)_{k}}{\left(b_{1}, \cdots, b_{s} ; q\right)_{k}}\left((-1)^{k} q^{\left(\begin{array}{c}
k \\
2
\end{array}\right)}\right)^{1+s-r} \frac{z^{k}}{(q ; q)_{k}},
$$


where

$$
\left(a_{1}, \cdots, a_{r}\right)_{k}:=\left(a_{1} ; q\right)_{k} \cdots\left(a_{r} ; q\right)_{k} .
$$

It is worth noting that the basic hypergeometric series fulfil the following identity also known as the $q$-binomial theorem

$$
{ }_{1} \phi_{0}\left(\begin{array}{c}
a \\
-
\end{array} \mid q ; z\right)=\sum_{n=0}^{\infty} \frac{(a ; q)_{n}}{(q ; q)_{n}} z^{n}=\frac{(a z ; q)_{\infty}}{(z ; q)_{\infty}}, \quad 0<|q|<1, \quad|z|<1 .
$$

Definition 2.4. The $q$-derivative operator is defined by

$$
D_{q} f(x)=\frac{f(q x)-f(x)}{(q-1) x}, \quad x \neq 0,
$$

and $D_{q} f(0)=f^{\prime}(0)$ provided that $f$ is differentiable at $x=0$.

The $q$-derivative fulfils the following product and quotient rules [15]

$$
\begin{aligned}
D_{q}(f(t) g(t)) & =f(q t) D_{q} g(t)+g(t) D_{q} f(t) . \\
D_{q}\left(\frac{f(t)}{g(t)}\right) & =\frac{g(q t) D_{q} f(t)-f(q t) D_{q} g(t)}{g(t) g(q t)} .
\end{aligned}
$$

Definition 2.5 ([7, page 36], see [15]). Suppose $0<a<b$. The definite $q$-integral is defined as

$$
\int_{0}^{b} f(x) d_{q} x=(1-q) b \sum_{n=0}^{\infty} q^{n} f\left(q^{n} b\right)
$$

and

$$
\int_{a}^{b} f(x) d_{q} x=\int_{0}^{b} f(x) d_{q} x-\int_{0}^{a} f(x) d_{q} x .
$$

Definition 2.6. The $q$-Gamma function is defined by

$$
\Gamma_{q}(x):=\frac{(q ; q)_{\infty}}{\left(q^{x} ; q\right)_{\infty}}(1-q)^{1-x}, \quad 0<q<1 .
$$

Remark 2.7. From Definition 2.6, the $q$-Gamma function is also represented by

$$
\Gamma_{q}(x)=(1-q)^{1-x}(q ; q)_{x-1} .
$$

Note also that the $q$-Gamma function satisfies the functional equation

$$
\Gamma_{q}(x+1)=[x]_{q} \Gamma_{q}(x), \quad \text { with } \quad \Gamma_{q}(1)=1 .
$$

For arbitrary complex $\alpha$,

$$
\left[\begin{array}{l}
\alpha \\
k
\end{array}\right]_{q}=\frac{\left(q^{-\alpha} ; q\right)_{k}}{(q ; q)_{k}}(-1)^{k} q^{\alpha k-\left(\begin{array}{c}
k \\
2
\end{array}\right) .}
$$

Or more generally, for all complex $\alpha$ and $\beta$ and $0<|q|<1$, we have

$$
\left[\begin{array}{c}
\alpha \\
\beta
\end{array}\right]_{q}:=\frac{\Gamma_{q}(\alpha+1)}{\Gamma_{q}(\beta+1) \Gamma_{q}(\alpha-\beta+1)}=\frac{\left(q^{\beta+1} ; q\right)_{\infty}\left(q^{\alpha-\beta+1} ; q\right)_{\infty}}{(q ; q)_{\infty}\left(q^{\alpha+1} ; q\right)_{\infty}} .
$$

Definition 2.8 ([15]). The $q$-Beta function is defined for $t, s>0$ by

$$
B_{q}(t, s)=\int_{0}^{1} x^{t-1}(1 \ominus q x)_{q}^{s-1} d_{q} x .
$$


It is worth noting that the $q$-Beta function and the $q$-Gamma function are related by [15, Eq (21.17)]

$$
B_{q}(t, s)=\frac{\Gamma_{q}(t) \Gamma_{q}(s)}{\Gamma_{q}(t+s)} .
$$

The following Cauchy product for infinite series applies

$$
\left(\sum_{n=0}^{\infty} A_{n}\right)\left(\sum_{n=0}^{\infty} B_{n}\right)=\sum_{n=0}^{\infty}\left(\sum_{k=0}^{n} A_{k} B_{n-k}\right) .
$$

In particular, if $A_{n}=\frac{a_{n} x^{n}}{[n]_{q} !}$ and $B_{n}=\frac{b_{n} x^{n}}{[n]_{q} !}$, then we have

$$
\left(\sum_{n=0}^{\infty} \frac{a_{n} x^{n}}{[n]_{q} !}\right)\left(\sum_{n=0}^{\infty} \frac{b_{n} x^{n}}{[n]_{q} !}\right)=\sum_{n=0}^{\infty}\left(\sum_{k=0}^{n}\left[\begin{array}{l}
n \\
k
\end{array}\right]_{q} a_{k} b_{n-k}\right) \frac{x^{n}}{[n]_{q} !} .
$$

\section{3. q-hypergeometric Bernoulli polynomials with one parameter}

The classical $q$-Bernoulli polynomials were introduced by Al Salam in [1] by the following generating function

$$
\frac{t e_{q}(x t)}{e_{q}(t)-1}=\sum_{n=0}^{\infty} B_{n}(x) \frac{t^{n}}{[n]_{q} !} .
$$

We introduce the $q$-hypergeometric Bernoulli polynomials by the following generating function

$$
\frac{t^{N} /[N]_{q} !}{e_{q}(t)-T_{N-1, q}(t)} e_{q}(x t)=\sum_{n=0}^{\infty} B_{n, q}(N, x) \frac{t^{n}}{[n]_{q} !}
$$

where

$$
T_{N, q}(t)=\sum_{n=0}^{N} \frac{t^{n}}{[n]_{q} !},
$$

and the $q$-hypergeometric Bernoulli numbers of the first kind by

$$
B_{n, q}(N)=B_{n, q}(N, 0),
$$

and they are generated by

$$
\frac{t^{N} /[N]_{q} !}{e_{q}(t)-T_{N-1, q}(t)}=\sum_{n=0}^{\infty} B_{n, q}(N) \frac{t^{n}}{[n]_{q} !} .
$$

Note that when $N=1$, we recover the $q$-Bernoulli polynomials (3.1).

We also introduce the $q$-hypergeometric Bernoulli polynomials of the second kind by the generating function

$$
\frac{t^{N} /[N]_{q} !}{E_{q}(t)-S_{N-1, q}(t)} E_{q}(x t)=\sum_{n=0}^{\infty} q^{\left(\begin{array}{c}
n \\
2
\end{array}\right)} \mathfrak{B}_{n, q}(N, x) \frac{t^{n}}{[n]_{q} !},
$$

where

$$
S_{N, q}(t)=\sum_{n=0}^{N} \frac{q^{\left(\begin{array}{c}
n \\
2
\end{array}\right)} t^{n}}{[n]_{q} !}
$$

and the $q$-hypergeometric Bernoulli numbers of the second kind by

$$
\mathfrak{B}_{n, q}(N)=\mathfrak{B}_{n, q}(N, 0),
$$

and they are generated by

$$
\frac{t^{N} /[N]_{q} !}{E_{q}(t)-S_{N-1, q}(t)}=\sum_{n=0}^{\infty} q^{\left(\begin{array}{c}
n \\
2
\end{array}\right)} \mathfrak{B}_{n, q}(N) \frac{t^{n}}{[n]_{q} !} .
$$


When $N=1,(3.3)$ reduces to (5.2) in [2] and (3.4) becomes (4.2) in the same paper. Note also that both (3.2) and (3.3) are $q$-analogues of (1.3).

It should be noted that in [18], the authors introduced the two-dimensional generalization of the Bernoulli polynomials $\mathcal{B}_{n, q}^{[m-1, \alpha]}(x, y)$ defined by the generating function

$$
\left(\frac{t^{m}}{e_{q}(t)-T_{m-1, q}(t)}\right)^{\alpha} e_{q}(t x) E_{q}(t y)=\sum_{n=0}^{\infty} \mathcal{B}_{n, q}^{[m-1, \alpha]}(x, y) \frac{t^{n}}{[n]_{q} !},
$$

and studied several of their properties. Another important generalization can be found in [21] but all these generalizations are different from ours.

3.1. The $q$-hypergeometric Bernoulli polynomials $B_{n, q}(N, x)$.

Proposition 3.1. The q-hypergeometric Bernoulli polynomials have the following representation

$$
B_{n, q}(N, x)=\sum_{k=0}^{n}\left[\begin{array}{l}
n \\
k
\end{array}\right]_{q} B_{k, q}(N) x^{n-k}=\sum_{k=0}^{n}\left[\begin{array}{l}
n \\
k
\end{array}\right]_{q} B_{n-k, q}(N) x^{k} .
$$

Proof. From definition (3.2), it follows that

$$
\begin{aligned}
\sum_{n=0}^{\infty} B_{n, q}(N, x) \frac{t^{n}}{[n]_{q} !} & =e_{q}(x t) \frac{t^{N} /[N]_{q} !}{e_{q}(t)-T_{N-1, q}(t)}=e_{q}(x t) \sum_{n=0}^{\infty} B_{n, q}(N) \frac{t^{n}}{[n]_{q} !} \\
& =\left(\sum_{n=0}^{\infty} x^{n} \frac{t^{n}}{[n]_{q} !}\right)\left(\sum_{n=0}^{\infty} B_{n, q}(N) \frac{t^{n}}{[n]_{q} !}\right) \\
& =\sum_{n=0}^{\infty}\left(\sum_{k=0}^{n}\left[\begin{array}{l}
n \\
k
\end{array}\right]_{q} B_{k, q}(N) x^{n-k}\right) \frac{t^{n}}{[n]_{q} !} .
\end{aligned}
$$

Proposition 3.2 ( $q$-analog of [10, Eq. (2.13)]). The following power representation holds

$$
x^{n}=[N]_{q} ! \sum_{k=0}^{n}\left[\begin{array}{l}
n \\
k
\end{array}\right]_{q} \frac{[n-k]_{q} !}{[N+n-k]_{q} !} B_{k, q}(N, x) .
$$

Proof. First, observe that

$$
\frac{e_{q}(t)-T_{N-1, q}(t)}{t^{N} /[N]_{q} !}=\frac{[N]_{q} !}{t^{N}} \sum_{n=N}^{\infty} \frac{t^{n}}{[n]_{q} !}=[N]_{q} ! \sum_{n=0}^{\infty} \frac{t^{n}}{[N+n]_{q} !} .
$$

From (3.2), it follows that

$$
\begin{aligned}
\sum_{n=0}^{\infty} x^{n} \frac{t^{n}}{[n]_{q} !} & =e_{q}(x t)=\frac{e_{q}(t)-T_{N-1, q}(t)}{t^{N} /[N]_{q} !} \sum_{n=0}^{\infty} B_{n, q}(N, x) \frac{t^{n}}{[n]_{q} !} \\
& =[N]_{q} !\left(\sum_{n=0}^{\infty} \frac{t^{n}}{[N+n]_{q} !}\right)\left(\sum_{n=0}^{\infty} B_{n, q}(N, x) \frac{t^{n}}{[n]_{q} !}\right) \\
& =[N]_{q} ! \sum_{n=0}^{\infty}\left(\sum_{k=0}^{n}\left[\begin{array}{l}
n \\
k
\end{array}\right]_{q} \frac{[n-k]_{q} !}{[N+n-k]_{q} !} B_{k, q}(N, x)\right) \frac{t^{n}}{[n]_{q} !} .
\end{aligned}
$$

The result follows by collecting the coefficients of $t^{n}$ on both sides.

Corollary 3.3. The following equation applies

$$
\sum_{k=0}^{n}\left[\begin{array}{l}
n \\
k
\end{array}\right]_{q} \frac{[n-k]_{q} !}{[N+n-k]_{q} !} B_{k, q}(N)=\left\{\begin{array}{ll}
\frac{1}{[N]_{q} !} & \text { if } n=0 \\
0 & \text { if } n>0
\end{array} .\right.
$$


Proof. Take $x=0$ in (3.6).

Theorem 3.4 (q-analog of [10, Eq. (2.16)]). Let $N \in \mathbb{N}^{*}$, the following equation holds for the q-hypergeometric Bernoulli polynomials

$$
\int_{0}^{1}(1 \ominus q x)_{q}^{N-1} B_{n, q}(N, x) d_{q} x=\frac{1}{[N]_{q}} \delta_{n, 0} .
$$

Proof. From (3.5), we have:

$$
\int_{0}^{1}(1 \ominus q x)_{q}^{N-1} B_{n, q}(N, x) d_{q} x=\sum_{k=0}^{n}\left[\begin{array}{l}
n \\
k
\end{array}\right]_{q} B_{k, q}(N) \int_{0}^{1}(1 \ominus q x)_{q}^{N-1} x^{n-k} d_{q} x .
$$

Using the definition of the $q$-Beta function given by (2.4) and Relation (2.5), the previous relation gives:

$$
\begin{aligned}
\int_{0}^{1}(1 \ominus q x)_{q}^{N-1} B_{n, q}(N, x) d_{q} x & =\sum_{k=0}^{n}\left[\begin{array}{l}
n \\
k
\end{array}\right]_{q} B_{k, q}(N) B_{q}(N, n-k+1) \\
& =\sum_{k=0}^{n}\left[\begin{array}{l}
n \\
k
\end{array}\right]_{q} B_{k, q}(N) \frac{[N-1]_{q} ![n-k]_{q} !}{[N+n-k]_{q} !} .
\end{aligned}
$$

From (3.7), it follows

$$
\int_{0}^{1}(1 \ominus q x)_{q}^{N-1} B_{n, q}(N, x) d_{q} x=[N-1]_{q} ! \sum_{k=0}^{n}\left[\begin{array}{c}
n \\
k
\end{array}\right]_{q} \frac{[n-k]_{q} !}{[N+n-k]_{q} !} B_{k, q}(N)=\frac{1}{[N]_{q}} \delta_{n, 0} .
$$

Proposition 3.5. The following equation holds

$$
D_{q} B_{n, q}(N, x)=[n]_{q} B_{n-1, q}(N, x) .
$$

Proof. First observe that

$$
D_{q}\left(e_{q}(x t)\right)=\sum_{n=0}^{+\infty} D_{q} x^{n} \frac{t^{n}}{[n]_{q} !}=t \sum_{n=1}^{+\infty} x^{n-1} \frac{t^{n-1}}{[n-1]_{q} !}=t e_{q}(x t) .
$$

Thus, we have

$$
\begin{aligned}
\sum_{n=0}^{+\infty} D_{q} B_{n, q}(N, x) \frac{t^{n}}{[n]_{q} !} & =t e_{q}(x t) \frac{t^{N} /[N]_{q} !}{e_{q}(t)-T_{N-1, q}(t)} \\
& =\sum_{n=0}^{+\infty} B_{n, q}(N, x) \frac{t^{n+1}}{[n]_{q} !} \\
& =\sum_{n=1}^{+\infty} B_{n-1, q}(N, x) \frac{t^{n}}{[n-1]_{q} !} \\
& =\sum_{n=1}^{+\infty}[n]_{q} B_{n-1, q}(N, x) \frac{t^{n}}{[n]_{q} !} .
\end{aligned}
$$

The result follows by identifying the coefficients of $t^{n}$. 
Theorem 3.6. The q-hypergeometric Bernoulli polynomials $B_{n, q}(N, x)$ are the only $q$ Appell polynomial set with zero moments, satisfying

$$
\begin{aligned}
B_{0, q}(N, x) & =1, \\
D_{q} B_{n, q}(N, x) & =[n]_{q} B_{n-1, q}(N, x), \\
\int_{0}^{1}(1 \ominus q x)_{q}^{N-1} B_{n, q}(N, x) d_{q} x & =\frac{1}{[N]_{q}} \delta_{n, 0} .
\end{aligned}
$$

Proof. It is already clear from equation (3.8) and Proposition 3.5 that the Bernoulli polynomials $B_{n, q}(N, x)$ satisfy (3.9), (3.10) and (3.11). Conversely, assume that a family of polynomials $P_{n, q}(N, x)$ satisfies (3.9), (3.10) and (3.11). By defining the series

$$
H(x, t)=\sum_{n=0}^{\infty} P_{n, q}(N, x) \frac{t^{n}}{[n]_{q} !} .
$$

From (3.10), it follows that $D_{q, x} H(x, t)=t H(x, t)$. Hence $H(x, t)=e_{q}(x t) h(t)$ where $h(t)$ is arbitrary unless additional constraints are given. It is now clear using the condition (3.11) that

$$
\begin{aligned}
\int_{0}^{1}(1 \ominus q x)^{N-1} H(x, t) d_{q} x & =\int_{0}^{1}(1 \ominus q x)^{N-1}\left(\sum_{n=0}^{\infty} P_{n, q}(N, x) \frac{t^{n}}{[n]_{q} !}\right) d_{q} x \\
& =\sum_{n=0}^{\infty} \frac{t^{n}}{[n]_{q} !} \int_{0}^{1}(1 \ominus q x)^{N-1} P_{n, q}(N, x) d_{q} x \\
& =\frac{1}{[N]_{q}} .
\end{aligned}
$$

The same integral can be computed otherwise in the following way

$$
\begin{aligned}
\int_{0}^{1}(1 \ominus q x)^{N-1} H(x, t) d_{q} x & =h(t) \int_{0}^{1}(1 \ominus q x)^{N-1} e_{q}(x t) d_{q} x \\
& =h(t) \int_{0}^{1}(1 \ominus q x)^{N-1} \sum_{n=0}^{\infty} \frac{(x t)^{n}}{[n]_{q} !} d_{q} x \\
& =h(t) \sum_{n=0}^{\infty} \frac{t^{n}}{[n]_{q} !} \int_{0}^{1}(1 \ominus q x)^{N-1} x^{n} d_{q} x \\
& =h(t) \sum_{n=0}^{\infty} \frac{t^{n}}{[n]_{q} !} \frac{\Gamma_{q}(n+1) \Gamma_{q}(N)}{\Gamma_{q}(N+n+1)} \\
& =[N-1]_{q} ! h(t) \sum_{n=0}^{\infty} \frac{t^{n}}{[N+n]_{q} !} \\
& =h(t)[N-1]_{q} ! \frac{e_{q}(t)-T_{N-1}(t)}{t^{N}} .
\end{aligned}
$$

Hence, we get

$$
h(t)=\frac{t^{N} /[N]_{q} !}{e_{q}(t)-T_{N-1}(t)}
$$

and so $P_{n, q}(N, x)=B_{n, q}(N, x)$.

Remark 3.7. Theorem 3.6 says that the $q$-hypergeometric Bernoulli polynomials can be defined by the three equations (3.9), (3.10) and (3.11). 
3.2. The $q$-hypergeometric Bernoulli polynomials $\mathfrak{B}_{n, q}(N, x)$.

Proposition 3.8. The q-hypergeometric Bernoulli polynomials of the second kind have the following representation

$$
\begin{aligned}
\mathfrak{B}_{n, q}(N, x) & =\sum_{k=0}^{n}\left[\begin{array}{l}
n \\
k
\end{array}\right]_{q} q^{k(k-n)} \mathfrak{B}_{k, q}(N) x^{n-k} \\
& =\sum_{k=0}^{n}\left[\begin{array}{l}
n \\
k
\end{array}\right]_{q} q^{k(k-n)} \mathfrak{B}_{n-k, q}(N) x^{k} .
\end{aligned}
$$

Proof. From Definitions (3.3) and (3.4), it follows that

$$
\begin{aligned}
\sum_{n=0}^{\infty} q^{\left(\begin{array}{c}
n \\
2
\end{array}\right)} \mathfrak{B}_{n, q}(N, x) \frac{t^{n}}{[n]_{q} !} & =E_{q}(x t) \frac{t^{N} /[N]_{q} !}{S_{q}(t)-S_{N-1, q}(t)}=E_{q}(x t) \sum_{n=0}^{\infty} q^{\left(\begin{array}{c}
n \\
2
\end{array}\right)} \mathfrak{B}_{n, q}(N) \frac{t^{n}}{[n]_{q} !} \\
& =\left(\sum_{n=0}^{\infty} q^{\left(\begin{array}{c}
n \\
2
\end{array}\right)} x^{n} \frac{t^{n}}{[n]_{q} !}\right)\left(\sum_{n=0}^{\infty} q^{\left(\begin{array}{c}
n \\
2
\end{array}\right)} \mathfrak{B}_{n, q}(N) \frac{t^{n}}{[n]_{q} !}\right) \\
& =\sum_{n=0}^{\infty} q^{\left(\begin{array}{c}
n \\
2
\end{array}\right)}\left(\sum_{k=0}^{n}\left[\begin{array}{l}
n \\
k
\end{array} q_{q}^{k(k-n)} \mathfrak{B}_{k, q}(N) x^{n-k}\right) \frac{t^{n}}{[n]_{q} !} .\right.
\end{aligned}
$$

The result follows by identifying the coefficient of $t^{n}$ on the both sides of the equation.

Proposition 3.9 (Second $q$-analog of [10, Eq. (2.13)]). The following power representation holds

$$
x^{n}=[N]_{q} ! \sum_{k=0}^{n}\left[\begin{array}{l}
n \\
k
\end{array}\right]_{q} q^{\left(\begin{array}{c}
k \\
2
\end{array}\right)+\left(\begin{array}{c}
n+N-k \\
2
\end{array}\right)-\left(\begin{array}{c}
n \\
2
\end{array}\right)} \frac{[n-k]_{q} !}{[N+n-k]_{q} !} \mathfrak{B}_{k, q}(N, x) .
$$

Proof. First observe that

$$
\frac{E_{q}(t)-S_{N-1, q}(t)}{t^{N} /[N]_{q} !}=\frac{[N]_{q} !}{t^{N}} \sum_{n=N}^{\infty} q^{\left(\begin{array}{c}
n \\
2
\end{array}\right)} \frac{t^{n}}{[n]_{q} !}=[N]_{q} ! \sum_{n=0}^{\infty} q^{\left(\begin{array}{c}
n+N \\
2
\end{array}\right)} \frac{t^{n}}{[N+n]_{q} !} .
$$

From (3.3), it follows

$$
\begin{gathered}
\sum_{n=0}^{\infty} q^{\left(\begin{array}{c}
n \\
2
\end{array}\right)} x^{n} \frac{t^{n}}{[n]_{q} !}=E_{q}(x t)=\frac{E_{q}(t)-S_{N-1, q}(t)}{t^{N} /[N]_{q} !} \sum_{n=0}^{\infty} q^{\left(\begin{array}{c}
n \\
2
\end{array}\right)} \mathfrak{B}_{n, q}(N, x) \frac{t^{n}}{[n]_{q} !} \\
=[N]_{q} !\left(\sum_{n=0}^{\infty} q^{\left(\begin{array}{c}
n+N \\
2
\end{array}\right)} \frac{t^{n}}{[N+n]_{q} !}\right)\left(\sum_{n=0}^{\infty} q^{\left(\begin{array}{c}
n \\
2
\end{array}\right)} \mathfrak{B}_{n, q}(N, x) \frac{t^{n}}{[n]_{q} !}\right) \\
=[N]_{q} ! \sum_{n=0}^{\infty} q^{\left(\begin{array}{c}
n \\
2
\end{array}\right)}\left(\sum_{k=0}^{n}\left[\begin{array}{l}
n \\
k
\end{array}\right]_{q} q^{\left(\begin{array}{c}
k \\
2
\end{array}\right)+\left(\begin{array}{c}
n+N-k \\
2
\end{array}\right)-\left(\begin{array}{c}
n \\
2
\end{array}\right)} \frac{[n-k]_{q} !}{[N+n-k]_{q} !} \mathfrak{B}_{k, q}(N, x)\right) \frac{t^{n}}{[n]_{q} !} .
\end{gathered}
$$

The result follows by collecting the coefficients of $t^{n}$ on both sides.

Corollary 3.10. The following equation applies

$$
\sum_{k=0}^{n}\left[\begin{array}{l}
n \\
k
\end{array}\right]_{q} q^{\left(\begin{array}{c}
k \\
2
\end{array}\right)+\left(\begin{array}{c}
n+N-k \\
2
\end{array}\right)-\left(\begin{array}{c}
n \\
2
\end{array}\right)} \frac{[n-k]_{q} !}{[N+n-k]_{q} !} \mathfrak{B}_{k, q}(N)=\frac{1}{[N]_{q} !} \delta_{n, 0} .
$$

Proposition 3.11. The following equation holds

$$
D_{q} \mathfrak{B}_{n, q}(N, x)=[n]_{q} \mathfrak{B}_{n-1, q}(N, q x) .
$$


Proof. Observe first that

$$
D_{q}\left(E_{q}(x t)\right)=t E_{q}(q x t) .
$$

Thus, we have

$$
\begin{aligned}
\sum_{n=0}^{+\infty} D_{q} \mathfrak{B}_{n, q}(N, x) \frac{t^{n}}{[n]_{q} !} & =t E_{q}(q x t) \frac{t^{N} /[N]_{q} !}{E_{q}(t)-T_{N-1, q}(t)} \\
& =\sum_{n=0}^{+\infty} \mathfrak{B}_{n, q}(N, q x) \frac{t^{n+1}}{[n]_{q} !} \\
& =\sum_{n=1}^{+\infty} \mathfrak{B}_{n-1, q}(N, q x) \frac{t^{n}}{[n-1]_{q} !} \\
& =\sum_{n=1}^{+\infty}[n]_{q} \mathfrak{B}_{n-1, q}(N, q x) \frac{t^{n}}{[n]_{q} !} .
\end{aligned}
$$

The result follows.

\section{The $q$-hypergeometric Bernoulli polynomials with two parameters}

Note that

$$
\begin{aligned}
\frac{e_{q}(t)-T_{N-1, q}(t)}{t^{N} /[N]_{q} !} & =\sum_{k=0}^{+\infty} \frac{[N]_{q} !}{[N+k]_{q} !} t^{k} \\
& =\sum_{k=0}^{+\infty} \frac{1}{\prod_{j=1}^{k}\left(1-q^{N+j}\right)}(1-q)^{k} t^{k} \\
& =\sum_{k=0}^{+\infty} \frac{(q ; q)_{k}(0 ; q)_{k}}{\left(q^{N+1} ; q\right)_{k}} \frac{(1-q)^{k} t^{k}}{(q ; q)_{k}} \\
& ={ }_{2} \phi_{1}\left(\begin{array}{c}
q, 0 \\
q^{N+1}
\end{array} \mid q,(1-q) t\right) .
\end{aligned}
$$

Thus, we observe that the generating function (3.2) can be expressed in terms of the basic hypergeometric function (see Definition 2.3)

$$
\frac{t^{N} e_{q}(x t) /[N]_{q} !}{e_{q}(t)-T_{N-1, q}(t)}=\frac{e_{q}(x t)}{{ }_{2} \phi_{1}\left(\begin{array}{c}
q, 0 \\
q^{N+1}
\end{array} \mid q,(1-q) t\right)}=\sum_{n=0}^{\infty} B_{n, q}(N, x) \frac{t^{n}}{[n]_{q} !}
$$

We therefore use (4.1) to define the $q$-hypergeometric Bernoulli polynomials in two continuous parameters $M$ and $N$ by

$$
\frac{e_{q}(x t)}{{ }_{2} \phi_{1}\left(\begin{array}{c}
q^{M+1}, 0 \\
q^{M+N+1}
\end{array} \mid q,(1-q) t\right)}=\sum_{n=0}^{\infty} B_{n, q}(M, N, x) \frac{t^{n}}{[n]_{q} !} .
$$

Obviously, for $M=0$ and $N$ a positive integer, we have $B_{n, q}(0, N, x)=B_{n, q}(N, x)$ where $B_{n, q}(N, x)$ is the $q$-hypergeometric Bernoulli polynomials defined by (3.2). These $q$ hypergeometric Bernoulli polynomials with two parameters are $q$-analogs of the Bernoulli polynomials in two parameters introduced in [6]. Also, we introduce the $q$-hypergeometric 
Bernoulli numbers in two parameters $B_{n, q}(M, N)=B_{n, q}(M, N, 0)$ by the generating function

$$
\frac{1}{{ }_{2} \phi_{1}\left(\begin{array}{c}
q^{M+1}, 0 \\
q^{M+N+1}
\end{array} \mid q,(1-q) t\right)}=\sum_{n=0}^{\infty} B_{n, q}(M, N) \frac{t^{n}}{[n]_{q} !} .
$$

Proposition 4.1. The following equation holds

$$
D_{q} B_{n, q}(M, N, x)=[n]_{q} B_{n-1, q}(M, N, x) .
$$

Proof. The proof is similar to the proof of Proposition 3.5.

Proposition 4.2. The q-hypergeometric Bernoulli polynomials with two parameters satisfy the following relation

$$
B_{n, q}\left(M, N,\left(x \oplus_{q} y\right)\right)=\sum_{k=0}^{n}\left[\begin{array}{l}
n \\
k
\end{array}\right]_{q} B_{k, q}(M, N, x) y^{n-k} .
$$

Proof. To prove (4.3), we first of all remark that the $q$-exponential function satisfies (see for example [16])

$$
e_{q}(x t) e_{q}(y t)=e_{q}\left(\left(x \oplus_{q} y\right) t\right)
$$

Using this equation, we have

$$
\begin{aligned}
\sum_{n=0}^{\infty} B_{n, q}\left(M, N,\left(x \oplus_{q} y\right)\right) \frac{t^{n}}{[n]_{q} !} & \frac{e_{q}\left(\left(x \oplus_{q} y\right) t\right)}{{ }_{2} \phi_{1}\left(\begin{array}{c}
q^{M+1}, 0 \\
q^{M+N+1}
\end{array} \mid q,(1-q) t\right)}=\frac{e_{q}(x t) e_{q}(y t)}{{ }_{2} \phi_{1}\left(\begin{array}{c}
q^{M+1}, 0 \\
q^{M+N+1}
\end{array} \mid q,(1-q) t\right)} \\
= & \left(\sum_{n=0}^{\infty} B_{n, q}(M, N, x) \frac{t^{n}}{[n]_{q} !}\right)\left(\sum_{n=0}^{\infty} y^{n} \frac{t^{n}}{[n]_{q} !}\right) \\
& \sum_{n=0}^{\infty}\left(\sum_{k=0}^{n}\left[\begin{array}{l}
n \\
k
\end{array}\right]_{q} B_{k, q}(M, N, x) y^{n-k}\right) \frac{t^{n}}{[n]_{q} !}
\end{aligned}
$$

This proves the proposition.

Corollary 4.3. The q-hypergeometric Bernoulli polynomials with two parameters satisfy the following relation

$$
B_{n, q}\left(M, N,\left(x \oplus_{q} 1\right)\right)-B_{n, q}(N, x)=\sum_{k=0}^{n-1}\left[\begin{array}{l}
n \\
k
\end{array}\right]_{q} B_{k, q}(N, x) .
$$

Proof. Take $y=1$ in (4.3).

Corollary 4.4. The q-hypergeometric Bernoulli polynomials with one parameter satisfy the following relation

$$
B_{n, q}\left(N,\left(x \oplus_{q} y\right)\right)=\sum_{k=0}^{n}\left[\begin{array}{l}
n \\
k
\end{array}\right]_{q} B_{k, q}(N, x) y^{n-k} .
$$

Proof. Take $M=0$ in (4.3).

Proposition 4.5. The q-hypergeometric Bernoulli polynomials with two parameters have the following explicit representation

$$
B_{n, q}(M, N, x)=\sum_{k=0}^{n}\left[\begin{array}{l}
n \\
k
\end{array}\right]_{q} B_{k, q}(M, N) x^{n-k} .
$$


Proof. The proof is similar to the proof of Proposition 3.1.

Proposition 4.6 ( $q$-analog of [10, Eq. (3.5)]). For real $M>0$ and $N>0$, the $q$ hypergeometric Bernoulli polynomials with two parameters satisfy the following representation

$$
x^{n}=\frac{\Gamma_{q}(M+N+1)}{\Gamma_{q}(M+1)} \sum_{k=0}^{n}\left[\begin{array}{l}
n \\
k
\end{array}\right]_{q} \frac{\Gamma_{q}(M+n-k+1)}{\Gamma_{q}(M+N+n-k+1)} B_{k, q}(M, N, x) .
$$

Proof. From the definition of the $q$-hypergeometric Bernoulli polynomials with two parameters (4.2), we have

$$
\begin{aligned}
& \sum_{n=0}^{\infty} x^{n} \frac{t^{n}}{[n]_{q} !}=e_{q}(x t)={ }_{2} \phi_{1}\left(\begin{array}{c}
q^{M+1}, 0 \\
q^{M+N+1}
\end{array} \mid q,(1-q) t\right)\left(\sum_{n=0}^{\infty} B_{n, q}(M, N, x) \frac{t^{n}}{[n]_{q} !}\right) \\
& =\left(\sum_{n=0}^{\infty} \frac{\left(q^{M+1} ; q\right)_{n}}{\left(q^{M+N+1} ; q\right)_{n}} \frac{t^{n}}{[n]_{q} !}\right)\left(\sum_{n=0}^{\infty} B_{n, q}(M, N, x) \frac{t^{n}}{[n]_{q} !}\right) \\
& =\sum_{n=0}^{\infty}\left(\sum_{k=0}^{n} \frac{B_{k, q}(M, N, x)}{[k]_{q} !} \frac{\left(q^{M+1} ; q\right)_{n-k}}{\left(q^{M+N+1} ; q\right)_{n-k}} \frac{1}{[n-k]_{q} !}\right) t^{n} \\
& =\sum_{n=0}^{\infty}\left(\frac{\Gamma_{q}(M+N+1)}{\Gamma_{q}(M+1)} \sum_{k=0}^{n}\left[\begin{array}{c}
n \\
k
\end{array}\right]_{q} \frac{\Gamma_{q}(M+n-k+1)}{\Gamma_{q}(M+N+n-k+1)} B_{k, q}(M, N, x)\right) \frac{t^{n}}{[n]_{q} !} .
\end{aligned}
$$

The result is obtained by identifying the coefficients of $t^{n}$ on the both sides of the previous equality.

Corollary 4.7. For real $M>0$ and $N>0$, the following equation applies

$$
\sum_{k=0}^{n}\left[\begin{array}{l}
n \\
k
\end{array}\right]_{q} \frac{\Gamma_{q}(M+n-k+1)}{\Gamma_{q}(M+N+n-k+1)} B_{k, q}(M, N)=\left\{\begin{array}{ll}
\frac{\Gamma_{q}(M+1)}{\Gamma_{q}(M+N+1)} & \text { if } n=0 \\
0 & \text { if } n>0
\end{array} .\right.
$$

Proof. Take $x=0$ in (4.5).

Theorem 4.8 (q-analog of [10, Eq. (3.3)]). For real $M>0$ and $N>0$, the following equation holds for the q-hypergeometric Bernoulli polynomials with two parameters

$$
\int_{0}^{1} x^{M-1}(1 \ominus q x)_{q}^{N-1} B_{n, q}(M, N, x) d_{q} x=\frac{\Gamma_{q}(N) \Gamma_{q}(M)}{\Gamma_{q}(M+N)} \delta_{n, 0} .
$$

Proof. From (4.4) and (4.6), if follows

$$
\begin{aligned}
\int_{0}^{1}(1 \ominus q x)_{q}^{N-1} x^{M-1} B_{n, q}(N, M, x) d_{q} x \\
=\sum_{k=0}^{n}\left[\begin{array}{l}
n \\
k
\end{array}\right]_{q} B_{k, q}(M, N) \int_{0}^{1}(1 \ominus q x)_{q}^{N-1} x^{M+n-k-1} d_{q} x \\
=\sum_{k=0}^{n}\left[\begin{array}{l}
n \\
k
\end{array}\right]_{q} \frac{\Gamma_{q}(N) \Gamma_{q}(M+n-k)}{\Gamma_{q}(M+N+n-k)} B_{k, q}(N, M) \\
=\Gamma_{q}(N) \sum_{k=0}^{n}\left[\begin{array}{l}
n \\
k
\end{array}\right]_{q} \frac{\Gamma_{q}(M+n-k)}{\Gamma_{q}(M+N+n-k)} B_{k, q}(N, M) \\
=\frac{\Gamma_{q}(N) \Gamma_{q}(M)}{\Gamma_{q}(M+N)} \delta_{n, 0} .
\end{aligned}
$$

Remark 4.9. Observe that (4.4) and (4.5) are inversions of each other. Moreover, Theorem 4.8 reduces to the $q$-beta function $B_{q}(M, N)$ for the case $n=0$. 


\section{Some multiplication formulas}

Proposition 5.1. Let a be a non zero complex number. The following equations apply

$$
\begin{aligned}
B_{n, q}(M, N, x) & =\sum_{k=0}^{n}\left[\begin{array}{l}
n \\
k
\end{array}\right]_{q}(a ; q)_{k} x^{k} B_{n-k}(M, N, a x) \\
a^{n} B_{n, q}\left(M, N, \frac{x}{a}\right) & =\sum_{k=0}^{n}\left[\begin{array}{l}
n \\
k
\end{array}\right]_{q}(a ; q)_{k} a^{n-k} x^{k} B_{n-k}(M, N, x) .
\end{aligned}
$$

Proof. From (4.2) and the $q$-binomial theorem (2.1), we get

$$
\begin{aligned}
\sum_{n=0}^{\infty} B_{n, q}(M, N, x) \frac{t^{n}}{[n]_{q} !} & =\frac{e_{q}(x t)}{{ }_{2} \phi_{1}\left(\begin{array}{c}
q^{M+1}, 0 \\
\left.q^{M+N+1} \mid q,(1-q) t\right)
\end{array}\right.} \\
& =\frac{e_{q}(a x t)}{{ }_{2} \phi_{1}\left(\begin{array}{c}
q^{M+1}, 0 \\
\left.q^{M+N+1} \mid q,(1-q) t\right)
\end{array}\right.} \\
& =\left(\sum_{n=0}^{\infty} B_{n, q}(M, N, a x) \frac{t^{n}}{e_{q}(a x]_{q} !}\right)\left(\sum_{n=0}^{\infty} \frac{(a ; q)_{n}}{[n]_{q} !} x^{n} t^{n}\right) \\
& =\sum_{n=0}^{\infty}\left(\sum_{k=0}^{n}\left[\begin{array}{l}
n \\
k
\end{array}\right]_{q}(a ; q)_{k} x^{k} B_{n-k}(M, N, a x)\right) \frac{t^{n}}{[n]_{q} !} .
\end{aligned}
$$

This proves (5.1). From (4.1) and the $q$-binomial theorem (2.1) again, we get

$$
\begin{aligned}
\sum_{n=0}^{\infty} B_{n, q}(M, N, x / a) \frac{t^{n}}{[n]_{q} !} & =\frac{e_{q}(x t / a)}{{ }_{2} \phi_{1}\left(\begin{array}{c}
q^{M+1}, 0 \\
q^{M+N+1}
\end{array} \mid q,(1-q) t\right)} \\
& =\frac{e_{q}(x t)}{{ }_{2} \phi_{1}\left(\begin{array}{c}
q^{M+1}, 0 \\
q^{M+N+1}
\end{array} \mid q,(1-q) t\right)} \frac{e_{q}(x t / a)}{e_{q}(x t)} \\
& =\left(\sum_{n=0}^{\infty} B_{n, q}(M, N, x) \frac{t^{n}}{[n]_{q} !}\right)\left(\sum_{n=0}^{\infty}(a ; q)_{n}\left(\frac{x}{a}\right)^{n} \frac{t^{n}}{[n]_{q} !}\right) \\
& =\sum_{n=0}^{\infty}\left(\frac{1}{a^{n}} \sum_{k=0}^{n}\left[\begin{array}{l}
n \\
k
\end{array}\right]_{q}(a ; q)_{k} a^{n-k} x^{k} B_{n-k}(M, N, x)\right) \frac{t^{n}}{[n]_{q} !} .
\end{aligned}
$$

Note that (5.1) reduces to [1, Eq (6.2)] where as (5.2) reduces to $[1,(6.3)]$ for $M=0$ and $N=1$.

Proposition 5.2. Let a be a non zero complex number. The following equations apply

$$
\begin{aligned}
& \mathcal{B}_{n, q}(N, x)=q^{-\left(\begin{array}{c}
n \\
2
\end{array}\right)} \sum_{k=0}^{n}\left[\begin{array}{l}
n \\
k
\end{array}\right]_{q} q^{\left(\frac{n-k}{2}\right)}(a \ominus 1)_{q}^{k} x^{k} \mathcal{B}_{n-k, q}(N, a x) \\
& \mathcal{B}_{n, q}\left(N, \frac{x}{a}\right)=q^{-\left(\begin{array}{c}
n \\
2
\end{array}\right)} \sum_{k=0}^{n}\left[\begin{array}{l}
n \\
k
\end{array}\right]_{q} q^{\left(\begin{array}{c}
n-k \\
2
\end{array}\right)}\left(a^{-1} ; q\right)_{k} x^{k} \mathcal{B}_{n-k, q}(N, x) .
\end{aligned}
$$


Proof. Let's define the function $H(t)=\frac{t^{N} /[N]_{q} \text { ! }}{E_{q}(t)-S_{N-1, q}(t)}$. Then from (3.3) and the identity (which follows from the $q$-binomial theorem $(2.1)$ )

$$
\frac{E_{q}(x t)}{E_{q}(a x t)}=\frac{((1-q) x t ; q)_{\infty}}{((1-q) a x t ; q)_{\infty}}=\sum_{n=0}^{\infty} \frac{(a \ominus 1)_{q}^{n}}{[n]_{q} !} x^{n} t^{n}
$$

it follows that

$$
\begin{aligned}
\sum_{n=0}^{\infty} q^{\left(\begin{array}{c}
n \\
2
\end{array}\right)} \mathcal{B}_{n, q}(N, x) \frac{t^{n}}{[n]_{q} !} & =H(t) E_{q}(x t)=H(t) E_{q}(a x t) \frac{E_{q}(x t)}{E_{q}(a x t)} \\
& =\left(\sum_{n=0}^{\infty} q^{\left(\begin{array}{c}
n \\
2
\end{array}\right)} \mathcal{B}_{n, q}(N, a x) \frac{t^{n}}{[n]_{q} !}\right)\left(\sum_{n=0}^{\infty} \frac{(a \ominus 1)_{q}^{n}}{[n]_{q} !} x^{n} t^{n}\right) \\
& =\sum_{n=0}^{\infty}\left(\sum_{k=0}^{n}\left[\begin{array}{l}
n \\
k
\end{array}\right]_{q} q^{\left(\begin{array}{c}
n-k \\
2
\end{array}\right)}(a \ominus 1)_{q}^{k} x^{k} \mathcal{B}_{n-k, q}(N, a x)\right) \frac{t^{n}}{[n]_{q} !}
\end{aligned}
$$

(5.3) follows. (5.4) follows from (5.3) by substituting $x$ by $\frac{x}{a}$.

\section{Recurrence relations and $q$-difference equations}

In this section we prove some recurrence relations and $q$-difference equations for the $q$ hypergeometric Bernoulli polynomials.

Lemma 6.1. If $G(x, t)=\frac{t^{N} /[N]_{q} !}{e_{q}(t)-T_{N-1}(t)} e_{q}(x t)$, then,

$$
t D_{q, t} G(x, t)=\left(q^{N} t x+[N]_{q}\right) G(x, t)-\left(t+[N]_{q} G(0, t)\right) G(x, q t) .
$$

Proof. Applying the product and the quotient rules (2.2) and (2.3) with $f(x, t)=\frac{t^{N}}{[N]_{q} !} e_{q}(x t)$ and $g(t)=e_{q}(t)-T_{N-1}(t)$, it follows that

$$
\begin{aligned}
D_{q, t} f(x, t) & =\frac{1}{[N]_{q} !}\left\{(q t)^{N} x e_{q}(x t)+[N]_{q} t^{N-1} e_{q}(x t)\right\} \\
& =\left\{q^{N} x+\frac{[N]_{q}}{t}\right\} f(x, t)
\end{aligned}
$$

and

Hence

$$
D_{q, t} g(t)=e_{q}(t)-T_{N-2}(t)=g(t)+\frac{t^{N-1}}{[N-1]_{q} !} .
$$

$$
D_{q, t} G(x, t)=\left\{q^{N} x+\frac{[N]_{q}}{t}\right\} G(x, t)-\left(1+\frac{t^{N-1}}{[N-1]_{q} !} \frac{1}{g(t)}\right) G(x, q t) .
$$

Theorem 6.2. The q-hypergeometric Bernoulli polynomials $B_{n, q}(N, x)$ are solutions of the following recurrence relation

$$
\begin{aligned}
\left([n]_{q}-[N]_{q}\right) B_{n, q}(N, x)= & {[n]_{q}\left(q^{N} x-q^{n-1}\right) B_{n-1, q}(N, x) } \\
& -[N]_{q} q^{n} \sum_{k=0}^{n}\left[\begin{array}{l}
n \\
k
\end{array}\right]_{q} B_{n-k, q}(N) B_{k, q}(N, x) .
\end{aligned}
$$


Proof. Observing that

$$
G(x, t)=\sum_{n=0}^{+\infty} B_{n, q}(N, x) \frac{t^{n}}{[n]_{q} !},
$$

the left-hand side of (6.1) gives

$$
t D_{q, t} G(x, t)=\sum_{n=0}^{+\infty}[n]_{q} B_{n, q}(N, x) \frac{t^{n}}{[n]_{q} !} .
$$

If we denote by $A$ the right side of (6.1), then we have

$$
\begin{aligned}
A= & q^{N} t \sum_{n=0}^{+\infty} x B_{n, q}(N, x) \frac{t^{n}}{[n]_{q} !}+[N]_{q} \sum_{n=0}^{+\infty} B_{n, q}(N, x) \frac{t^{n}}{[n]_{q} !}-\sum_{n=0}^{+\infty} B_{n, q}(N, x) q^{n} \frac{t^{n+1}}{[n]_{q} !} \\
& -[N]_{q}\left(\sum_{n=0}^{+\infty} B_{n, q}(N) q^{n} \frac{t^{n}}{[n]_{q} !}\right)\left(\sum_{n=0}^{+\infty} B_{n, q}(N, x) q^{n} \frac{t^{n}}{[n]_{q} !}\right) \\
= & q^{N} \sum_{n=0}^{+\infty}[n]_{q} x B_{n-1, q}(N, x) \frac{t^{n}}{[n]_{q} !}+[N]_{q} \sum_{n=0}^{+\infty} B_{n, q}(N, x) \frac{t^{n}}{[n]_{q} !}-\sum_{n=0}^{+\infty}[n]_{q} q^{n-1} B_{n-1, q}(N, x) \frac{t^{n}}{[n]_{q} !} \\
& -[N]_{q} \sum_{n=0}^{+\infty}\left(\sum_{k=0}^{n}\left[\begin{array}{c}
n \\
k
\end{array}\right]_{q} B_{n-k, q}(N) q^{n-k} B_{k, q}(N, x) q^{k}\right) \frac{t^{n}}{[n]_{q} !} .
\end{aligned}
$$

Hence, the right side of (6.1) gives

$$
\begin{aligned}
A= & \sum_{n=0}^{+\infty}\left(q^{N}[n]_{q} x B_{n-1, q}(N, x)+[N]_{q} B_{n, q}(N, x)-[n]_{q} q^{n-1} B_{n-1, q}(N, x)\right. \\
& \left.-[N]_{q}\left(\sum_{k=0}^{n}\left[\begin{array}{l}
n \\
k
\end{array}\right]_{q} B_{n-k, q}(N) q^{n-k} B_{k, q}(N, x) q^{k}\right)\right) \frac{t^{n}}{[n]_{q} !} .
\end{aligned}
$$

Considering the fact that $(6.2)=(6.3)$, we get the proof of the Theorem.

Theorem 6.3. The q-hypergeometric Bernoulli polynomials are solutions of the following q-differential equation

$$
\begin{aligned}
\left(q^{N} x-q^{n-1}\right) D_{q} B_{n, q}(N, x)= & \left([n]_{q}-[N]_{q}\right) B_{n, q}(N, x) \\
& +\sum_{k=0}^{n} \frac{[N]_{q} q^{n}}{[k]_{q} !} B_{k, q}(N) D_{q}^{k} B_{n, q}(N, x) .
\end{aligned}
$$

Proof. Using Theorem 6.2 and Proposition 3.5, we obtain the result.

\section{7. q-hypergeometric Bernoulli polynomials of higher order}

Let $r$ be an arbitrary complex number. We define the $q$-hypergeometric Bernoulli polynomials of order $r$ by

$$
\frac{e_{q}(x t)}{\left[{ }_{2} \phi_{1}\left(\begin{array}{c}
q^{M+1}, 0 \\
q^{M+N+1}
\end{array} \mid q,(1-q) t\right)\right]^{r}}=\sum_{n=0}^{\infty} B_{n, q}^{(r)}(M, N, x) \frac{t^{n}}{[n]_{q} !},
$$

and the $q$-hypergeometric Bernoulli numbers of order $r$ by the generating functions

$$
\frac{1}{\left[{ }_{2} \phi_{1}\left(\begin{array}{c}
q^{M+1}, 0 \\
q^{M+N+1}
\end{array} \mid q,(1-q) t\right)\right]^{r}}=\sum_{n=0}^{\infty} B_{n, q}^{(r)}(M, N) \frac{t^{n}}{[n]_{q} !},
$$


Proposition 7.1. The q-hypergeometric Bernoulli polynomials of order $r$ fulfil the following representation

$$
B_{n, q}^{(r)}(M, N, x)=\sum_{k=0}^{n}\left[\begin{array}{l}
n \\
k
\end{array}\right]_{q} B_{n-k, q}^{(r)}(M, N) x^{k} .
$$

Proof. From (7.1) and (7.2), it follows that

$$
\begin{aligned}
\sum_{n=0}^{\infty} B_{n, q}^{(r)}(M, N, x) \frac{t^{n}}{[n]_{q} !} & =\frac{e_{q}(x t)}{\left[{ }_{2} \phi_{1}\left(\begin{array}{c}
q^{M+1}, 0 \\
q^{M+N+1}
\end{array} \mid q,(1-q) t\right)\right]^{r}} \\
& =\left(\sum_{n=0}^{\infty} B_{n, q}^{(r)}(M, N) \frac{t^{n}}{[n]_{q} !}\right)\left(\sum_{n=0}^{\infty} x^{n} \frac{t^{n}}{[n]_{q} !}\right) \\
& =\sum_{n=0}^{\infty}\left(\sum_{k=0}^{n}\left[\begin{array}{l}
n \\
k
\end{array}\right]_{q} B_{n-k, q}^{(r)}(M, N) x^{k}\right) \frac{t^{n}}{[n]_{q} !}
\end{aligned}
$$

Let's define the function $F_{r}(t)=\frac{1}{\left[{ }_{2} \phi_{1}\left(\begin{array}{c}q^{M+1}, 0 \\ q^{M+N+1}\end{array} \mid q,(1-q) t\right)\right]^{r}}$ and $G_{r}(x, t)=F_{r}(t) e_{q}(x t)$.

It is obvious that $G_{r}(x, t)$ generates the $q$-hypergeometric Bernoulli polynomials of order $r$ whereas $F_{r}(t)$ generates the $q$-hypergeometric Bernoulli numbers of order $r$.

Proposition 7.2. The following q-addition formula holds

$$
B_{n, q}^{(r+s)}\left(M, N,\left(x \oplus_{q} y\right)\right)=\sum_{k=0}^{n}\left[\begin{array}{l}
n \\
k
\end{array}\right]_{q} B_{k, q}^{(r)}(M, N, x) B_{n-k}^{(s)}(M, N, y) .
$$

Proof. The proof follows from the equation $G_{r+s}\left(\left(x \oplus_{q} y\right), t\right)=G_{r}(x, t) G_{r}(y, t)$.

Corollary 7.3. The following equation applies

$$
\left(x \oplus_{q} y\right)^{n}=\sum_{k=0}^{n}\left[\begin{array}{l}
n \\
k
\end{array}\right]_{q} B_{k, q}^{(r)}(M, N, x) B_{n-k}^{(-r)}(M, N, y)
$$

Corollary 7.4. The following equation applies

$$
B_{n, q}^{(r+s)}(M, N, x)=\sum_{k=0}^{n}\left[\begin{array}{l}
n \\
k
\end{array}\right]_{q} B_{n-k}^{(s)}(M, N) B_{k, q}^{(r)}(M, N, x) .
$$

For example, for $s=1$, we get

$$
B_{n, q}^{(r+1)}(M, N, x)=\sum_{k=0}^{n}\left[\begin{array}{l}
n \\
k
\end{array}\right]_{q} B_{n-k}(M, N) B_{k, q}^{(r)}(M, N, x) .
$$

\section{Conclusion and further remarks}

In this paper we have introduced two kinds of $q$-hypergeometric Bernoulli polynomials that generalized the hypergeometric Bernoulli polynomials introduced in [6], extensively studied in $[10,14]$ and the reference therein. Classical results are obtained from the results of this paper by taking the limit as $q$ tends to 1 . These new families of $q$-Bernoulli polynomials will probably find applications in many areas of applied mathematics.

Acknowledgment. We would like to thank the anonymous reviewers whose valuable comments and remarks helped a lot to improve the manuscript. 


\section{References}

[1] W.A. Al-Salam, q-Bernoulli numbers and polynomials, Math. Nachr. 17, 239-260, 1959.

[2] W.A. Al-Salam, q-Appell polynomials, Ann. Mat. Pura Appl. 77 (4), 31-45, 1967.

[3] P. Appell, Une classe de polynomes, Annalles scientifique, Ecole Normale Sup., Ser. $2, \mathbf{9}, 119-144,1880$.

[4] F. Avram and M.S. Taqqu, Noncentral limit theorems and Appell polynomials, Ann. Probab. 15, 767-775, 1987.

[5] L. Comtet, Advanced Combinatorics, Reidel, Dordrecht, 1974.

[6] K. Dilcher, Bernoulli numbers and confluent hypergeometric functions, in: Number Theory for the Millennium, I (Urbana, IL, 2000), 343-363, A K Peters, Natick, MA, 2002.

[7] M. Foupouagnigni, Laguerre Hahn Orthogonal Polynomials with respect to the Hahn Operator, Fourth-order Difference Equation for the rth Associated and the LaguerreFreud Equations for the Recurrence Coefficients [dissertation]. Porto Novo: Université Nationale du Benin, ISMP, 1998.

[8] G. Gasper and M. Rahman, Basic hypergeometric Series, Encyclopedia Math. Appl. 35, Cambridge Univ. Press, Cambridge, 1990.

[9] R.L. Graham, D.E. Knuth and O. Patashnik, Concrete Mathematics, second ed., Addison-Wesley, Reading, 1994

[10] A. Hassen and H.D. Nguyen, Hypergeometric Bernoulli polynomials and Appell sequences, Int. J. Number Theory 4, 767-774, 2008.

[11] G. Hetyei, Enumeration by kernel positions for strongly Bernoulli type truncation games on words, J. Combin. Theory Ser. A, 117, 1107-1126, 2010.

[12] F.T. Howard, A sequence of numbers related to the exponential function, Duke Math. J. 34, 599-616, 1967.

[13] F.T. Howard, Some sequences of rational numbers related to the exponential function, Duke Math. J. 34, 701-716, 1967.

[14] S. Hu and M.-S. Kim, On hypergeometric Bernoulli numbers and polynomials, Acta Math. Hungar. 154, 134-146, 2018.

[15] V. Kac and P. Cheung, Quantum calculus, Springer, 2001.

[16] T. Kim, q-Extension of the Euler Formula and Trigometric functions, Russ. J. Math. Phys. 13 (3), 275-278, 2007.

[17] R. Koekoek, P.A. Lesky and R.F. Swarttouw, Hypergeometric Orthogonal Polynomials and their q-Analogues, Springer, Berlin, 2010.

[18] N.I. Mahmudov and M.E. Keleshteri, On a class of generalized q-Bernoulli and qEuler polynomials, Adv. Differ. Equ. 2013, Article number: 115, 2013.

[19] H. Pan and Z.-W. Sun, New identities involving Bernoulli and Euler polynomials, J. Combin. Theory Ser. A 113, 156-175, 2006.

[20] P. Tempesta, Formal groups, Bernoulli-type polynomials and L-series, C. R. Math. Acad. Sci. Paris 345, 303-306, 2007.

[21] A. Urieles, M.J. Ortega, W. Ramirez and S. Vega, New result on the q-generalized Bernoulli polynomials of level $m$, Demonstr. Math. 52, 511-522, 2019. 